\title{
Self-Regulating Plant Robots: Bioinspired Heliotropism and Nyctinasty
}

\author{
Suleyman Doruk Cezan, ${ }^{1}$ Hasan Tarik Baytekin,' and Bilge Baytekin ${ }^{1,2}$
}

\begin{abstract}
Self-regulation (or so-called homeostasis) is a property of all living organisms to maintain an internal stable state through specialized biofeedback mechanisms under varying external and internal conditions. Although these feedback mechanisms in living organisms are complex networks and hard to implement one-to-one in artificial systems, the new approaches in soft robotics may benefit from the concept of self-regulation-especially in the new endeavors of making untethered, autonomous soft robots. In this study, we show a simple system, in which plant robots display heliotropism (sun tracking) and nyctinasty (leaf opening) through artificial self-regulation attained through a bioinspired transpiration mechanism. The feedback involves dehydration/hydration and transpiration events that keep the stem continuously in a metastable position, which maximizes light on plant leaves and the efficiency of light harvesting when solar panels are attached on leaves. We also demonstrate that this artificial feedback can be regulated by doping with light-absorbing chemicals or by changing the geometry of the system, and it can further be expanded to other lightweight systems. Implementing self-regulation into (soft) robots through bioinspired material feedback is beneficial not only for energy efficiency and harvesting but also for achieving embodied intelligence in autonomous soft robots.
\end{abstract}

Keywords: embodied intelligence, material feedback, plant robots, artificial heliotropism, and nyctinasty

\section{Introduction}

T HE NEXT EPISODE IN SOFT ROBOTICS, robotic systems with agility, flexibility, and function that are inspired by living organisms, involves the design of untethered autonomous systems. ${ }^{1,2}$ Once seen as a "mission impossible," the autonomous soft robots are now emancipating themselves from their tethers, as shown by a few recent examples. ${ }^{3-5}$ One way to accomplish such autonomy and untethering is to implement self-regulation in robotics, similar to the frequently encountered biochemical feedback in living organisms (Fig. 1a). Although there are some recent studies on bioinspired selfhealing ${ }^{6}$ and other examples of materials and synthetic systems using chemical feedback, ${ }^{7}$ the use of self-regulation ${ }^{8}$ or material feedback in robotics has not been practiced yet, except in our recent study on artificial heliotropism and nyctinasty in plant robots with material feedback. ${ }^{5}$

We purposively constructed a robot resembling a plant in our previous study. ${ }^{5}$ Plants display many sophisticated and versatile movements that have astonished and inspired scientists and engineers for many centuries. ${ }^{9}$ Plants are the masters of energy efficiency and flexibility, which are the primary elements for soft robot design. The low number of reports on plant-inspired robots in comparison with the abundance of the reports on animal-inspired robots stays as a striking contrast to plants' resourcefulness in providing inspiration to many synthetic materials and systems. Nevertheless, in literature, there are some ingenious examples of plant-inspired robots such as the ones using osmotic actuation $^{10,11}$ or sensory behavior. ${ }^{12}$

Plant movements initiated and directed by external stimuli are particularly influential in designing artificial systems, such as sun trackers for efficient harvesting of solar ener$\mathrm{gy}^{5,13}$ or artificial root systems. ${ }^{14}$ We have previously selected two of these movements, heliotropism (a tropic movement) and nyctinasty (a nastic movement) ${ }^{15}$ for designing a robotic system that displays self-regulation. Although our previous design successfully displayed both of

${ }^{1}$ Department of Chemistry, Bilkent University, Ankara, Turkey.

${ }^{2}$ UNAM-Materials Science and Nanotechnology Institute, Bilkent University, Ankara, Turkey. 
these movements, it failed to be a real soft robot since the structural (plastics) and functional (nitinol springs) elements were all of hard materials. In addition, this previous system provided the necessary self-regulation through the nitinol thermomechanical hysteresis loop, which does not mimic biological mechanisms.

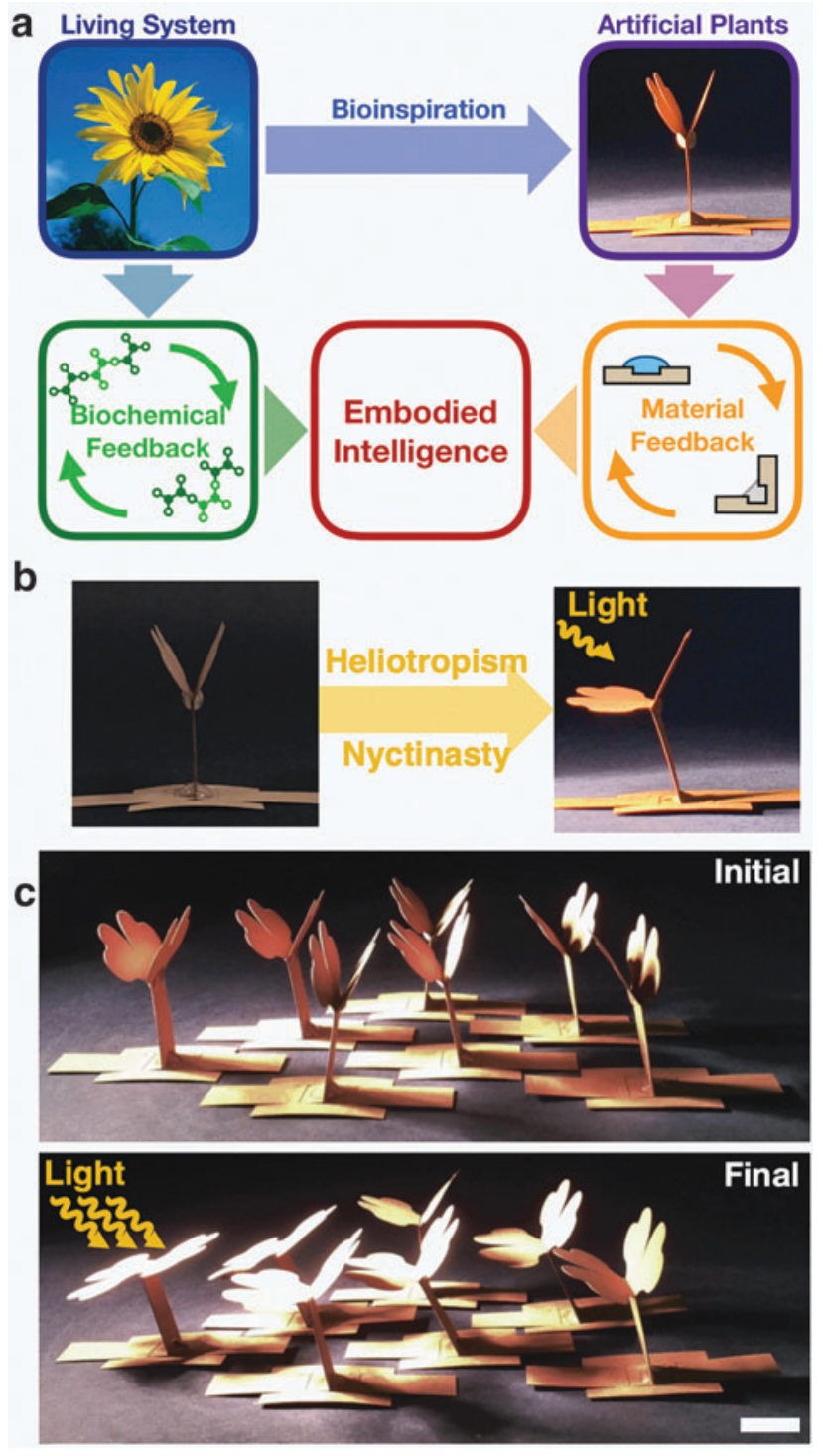

FIG. 1. Self-regulation and embodied intelligence in origami/ kirigami paper plant robots. (a) Self-regulation by biochemical feedback leads to embodied intelligence in living systems. A similar embodied intelligence can be achieved in (plant) robots through material feedback. (b) Smart arrangement of paper-gel muscles on the plant robot allows material feedback. Left: The plant in the dark (initial state of the plant), with its leaves closed. Right: Upon light input, the leaves open (nyctinasty) and the stem bends toward the light source (heliotropism). Final nonequilibrium state of the plant persists as long as the light input exists. When the plant is connected to a water reservoir, both motions are regulated autonomously through material feedback (Fig. 4). See Supplementary Movie S1. (c) A field of robot plants showing a gradual response according to their position with respect to the light source, displaying agile continuous action (as expected from soft robots) controlled by the intensity of the light stimulus. See Supplementary Movie S2. Scale bars are $1 \mathrm{~cm}$. Color images are available online.
In this study, we show an all-soft system, which displays similar self-regulation through heliotropism and nyctinasty upon light (infrared, IR) stimulus-but this time, the system mimics the living plant: the plant motion is achieved by swelling/deswelling of hydrogels attached at the joints of artificial plants. In our artificial plants, feedback is attained by transpiration, which is the water transport mechanism in plants. Although several other plant-inspired mechanisms such as (bio)chemical ${ }^{15}$ actuation can be utilized for actuating the robot, in our self-regulated system, we choose simple water transpiration since (1) water is the most significant element of growth, metabolism, and diurnal motion of plants, (2) both heliotropism and nyctinasty are performed by changing the turgor pressure of plant cells depending on light illumination, ${ }^{16}$ and (3) various types of motions performed by plants toward or away from environmental stimuli (such as light, heat, chemicals, humidity, gravity, electric field, temperature, and touch) ${ }^{9}$ occur by the essential contribution of transpiration, which allows elaboration of the current system to more complex future systems.

We also refrain ourselves from using more sophisticated materials such as liquid crystal elastomers, which have shown superior thermoresponsive behavior in similar systems ${ }^{17-21}$; however, they require many synthetic steps and complex sample handling. In this report, we also show that some plant parts, for example, a stoma opening, which may be integrated into soft robots, can also be actuated in a similar reversible manner and some mechanical metamaterial designs (auxetics) can also profit from the same approach. ${ }^{22-24}$ Although our (plant) systems cannot compete with average actuation speeds of current soft robotic systems displaying locomotion, they operate in the timescale for biological heliotropism/nyctinasty events (Supplementary Movie S1).

\section{Materials and Methods}

\section{Turgor-mediated plant movement and transpiration}

Plants rely on other mechanisms than muscles for movement. ${ }^{25}$ In plants that display heliotropic action, movement was found to be either growth mediated or turgor mediated. ${ }^{26,27}$ The latter is a reversible process that involves elastic expansion or contraction of plants' leaf cells (in specialized tissues called pulvinus) as a result of changes in turgor pressure in the cells. These changes are regulated by active and passive transport of solutes through the cell membranes. The anisotropy in the volume changes in cells accommodated in opposite sides of the pulvinus tissue results in a net movement by curvature. ${ }^{27}$ Since turgor-mediated movement is completely reversible, repeatable, and more straightforward than a growth-mediated mechanism to establish in an artificial system, we use swelling/deswelling of hydrogel actuators to resemble this mechanism in our artificial plants.

In our plants, the gel actuators are powered and controlled by a much simpler mechanism than the bio-hydroelectric motor $^{27}$ (solute transportation by membrane proteins to regulate the water potential across the membrane) of real plants. Instead, we coupled the swelling/deswelling of actuators to a simple water transport mechanism (transpiration). Transpiration also exists in real plants as a means of water transport between the roots of the plant and evaporation from the plant's aerial parts such as leaves, flowers, and stem. ${ }^{28,29}$ Since the plants constitute the largest living mass on earth, ${ }^{30}$ 
one might think about transpiration as the most widely used water transport mechanism on earth. ${ }^{31}$ Plants use transpiration to regulate their temperature and osmotic pressure in cells.

Transpiration occurs through capillary action and the driving force for it is the chemical potential difference of water in different parts of the plant. It is controlled by nonequilibrium feedback, in which water is continuously pumped from the roots through the plant body as the leaves and stem evaporate water-in a large tree, the amount of water transpirated may reach tons per day. ${ }^{15,32,33}$ As we show below, we use artificial transpiration as the continuous input that is necessary to maintain a nonequilibrium feedback in our artificial plant.

\section{Choice of materials}

To make an artificially heliotropic plant robot, we first developed a plant-inspired robotic body that performs the water transport function of the cellulose/lignin composite network in plants. ${ }^{34}$ In our earlier designs, we had used threedimensional, printed acrylonitrile-butadiene-styrene and polystyrene robot bodies with channels designed for water transport; however, in the final architectures, we switched to cellulose paper, which has intrinsic hydrophilicity. ${ }^{35}$ Using paper - a natural, biodegradable easily available materialas the body material also provided straightforward manufacture through cutting/engraving. Therefore, we prepared the first artificial plants by using origami and kirigami strategies on cellulose paper (Canson $1557-200 \mathrm{~g} / \mathrm{m}^{2}$, for details of the design and production, see Supplementary Fig. S1).

Although many similar types of paper can be used to obtain similar results, we preferred especially this type of thick, durable cellulose paper to engrave the crease patterns, which enables tens of reversible operation cycles without loss of reversibility. We also chose simple, general-use filter paper to serve as the roots and to transport water from the roots to the actuators. To serve as the actuator, we selected common hydrogels that resemble the soft water-filled tissues of plants. In literature, there are numerous reports on hydrogels that can be used to mimic the sensory behavior/motion and actuation in robotics. ${ }^{36-40}$ In this case, among a few examples that we tried [agarose, gelatin, polyacrylamide, and thermoresponsive poly( $\mathrm{N}$-isopropylacrylamide) gel], we found that the agarose gel performs the best (hydration/dehydration rates, sticking on the paper, and reversible hydration) for actuation through a simple transpiration mechanism.

With these materials and manufacturing method, a plant robot that can display heliotropism and nyctinasty (Fig. 1b) can be manufactured within 1 min after preparation of hydrogels and engraving of the paper, and a plant garden can be obtained in about $10 \mathrm{~min}$ - in which many plants simultaneously operate upon a single light input (Fig. 1c; Supplementary Movie S2).

\section{Results}

\section{Reversible actuation of hydrogel/paper actuators}

To build a transpiration-controlled feedback actuator, designated areas on the cellulose paper are cut and engraved by a laser cutter (Universal VLS2.30 model) to form the grooves for deposition of hydrogel. The actuation can be displayed in a simple actuator formed on a single-crease paper $(5 \times 30 \times 0.5 \mathrm{~mm})$ doped with $10-50 \mu \mathrm{L}$ of the $1-3 \%$ (w/w) agarose gel (Sigma-Aldrich) onto the engraved creased area (Supplementary Fig. S2a). When the gel is hydrated, the paper-gel actuator stays flat (swollen state). However, when the gel is dehydrated, the paper-gel actuator starts bending depending on the amount of water left in the gel (contracted state) (Supplementary Fig. S2b).

The dehydration (bending) actuation can be achieved by illuminating with a lamp (IR, 250W; General Electric) (Supplementary Fig. S2c) and is fully reversible upon hydration with water input from a connected reservoir, which continuously supplies water to the gel through the paper stem. As shown in Supplementary Fig. S3 and Supplementary Movie S3, the actuator and the artificial plant work essentially similarly under the sun outdoors; however, since the laboratory tests with an artificial light source are more reliable and reproducible, we conducted our experiments under laboratory conditions to reduce errors arising from different environmental conditions, for example, wind. We used an IR camera (Fluke TiX560) to monitor and compare the speed of water transport in various types of papers with different hydrophilicity, for example, for the Canson paper/filter paper system, $\sim 10 \mathrm{~s}$ is needed for water to travel $5 \mathrm{~cm}$, as shown in Supplementary Figure S1.

The timescale of the overall hydration/dehydration cycles in the actuator shown in Supplementary Fig. S4 significantly depends upon the gel concentration and its amount and was found to be in the order of tens of seconds for 3\% agarose gel on the Canson paper/filter paper system. Relative humidity (RH) is also found to be important for the speed of the change in folding angle. When hydrogel actuators are operated under dry conditions $(\mathrm{RH}=20 \%)$, faster response times were observed compared with the speed of operation at higher $\mathrm{RH}$ (90\%) (Supplementary Fig. S5). Dehydration can be accelerated by the addition of well-known IR light absorbers such as graphite (Supplementary Fig. S6). ${ }^{41}$ The average force obtained by such an actuator is a few tens of $\mathrm{mNs}$ (Supplementary Fig. S7).

The gel actuator can also be made to work in various directions by tuning the crease angle with respect to the short axis of the paper piece, as shown in Supplementary Figure S8. The actuators can also be combined in series to show a sequential bending of paper, which can be achieved upon illumination (Supplementary Fig. S9).

\section{Heliotropic plants}

We first describe a simple design for heliotropism only. A design shown in Supplementary Figure S10 is cut and engraved by a laser cutter and folded into the final shape, as shown in Figure 2. This design has two back-to-back actuators at the bottom of the stem, which is formed by applying 10-50 $\mu \mathrm{L}$ of gel on the crease areas marked with $\mathrm{H}$ in Figure 2. In the design for heliotropism only, the leaves, which cast shadows on the gels, stay open at all times. At the initial (dark) state, the plant stands upright, and the two actuators are bent at $90^{\circ}$ (Fig. 2a). When the light (IR lamp, distance to plant $50 \mathrm{~cm}$ ) is turned on and the elevation angle of the light source $\alpha$ is $90^{\circ}$, shadows of leaves fall on the two gel actuators and the plant keeps its upright position.

In cases where the light source's elevation is low (where $\alpha \neq 90^{\circ}$ ), one of the two gel actuators is illuminated and heated, as shown by the IR images in Figure $2 b$, which causes its dehydration. This actuator contracts in a few minutes (depending on the intensity of the incident light, 2500-5000 Lux) 

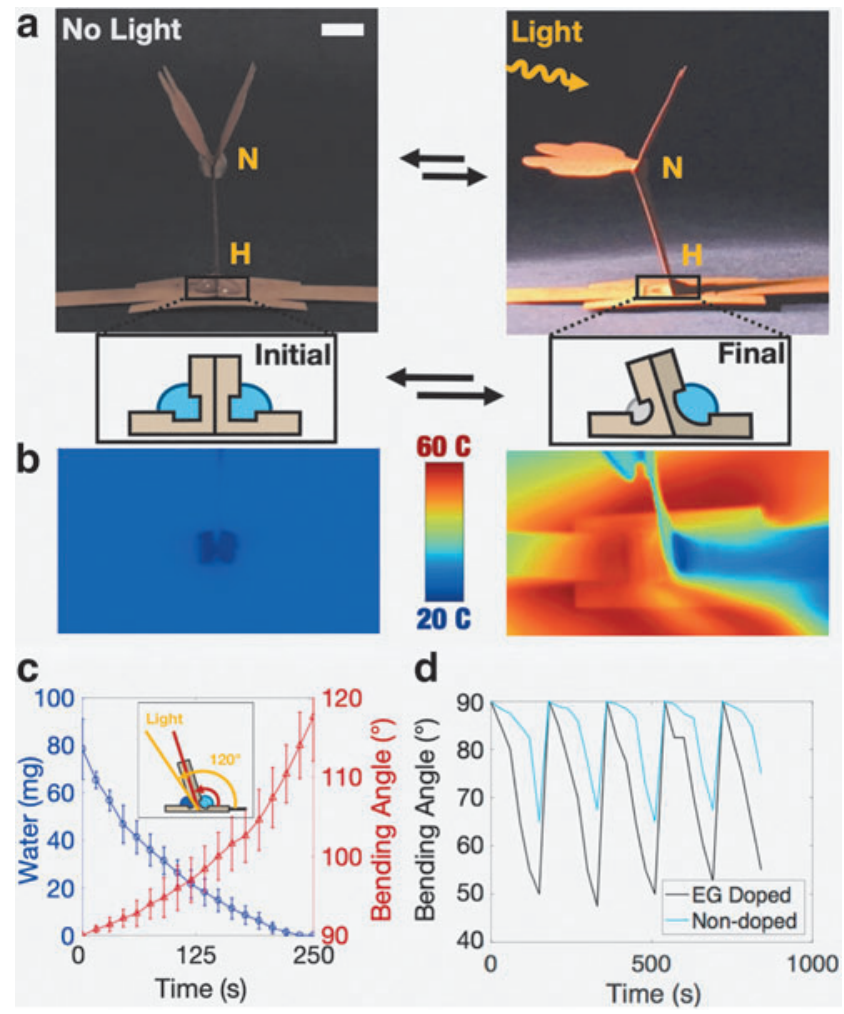

FIG. 2. The plant robot operated by transpiration showing heliotropism. (a) Visible and (b) thermal images of the robot operation; when the plant in the dark steady state (left) is exposed to light, water evaporates faster from the hydrogelpaper muscle on the illuminated and warmer side closer to the light source - the stem bends toward the light source, until the leaves cast shadows on the muscle. Scale bar $=1 \mathrm{~cm}$. (c) The heliotropic behavior, adaptive bending of the plant at different elevation angles (red), is attained through transpiration (water loss, blue) controlled by shadow casting. (d) Adding infrared heaters into the hydrogel (data shown for EG) increases the speed of heliotropic action without loss of reversibility. EG, exfoliated graphite. Color images are available online.

as a result of dehydration, which leads the stem to tilt toward the light source, until shadows of leaves fall on the gel actuator. When the shadow covers the gel, heating is interrupted, loss of water ceases (Fig. 2c), and the heliotropic motion stops at that bending angle (Supplementary Movie S1). This actuation is fully reversible, and rehydration takes place in a few minutes in the dark if the plant is connected to a reservoir of water.

The two-actuator plant robot we described here is the simplest design to display heliotropism. With proper chemicals, the efficiency of this design can be enhanced, that is, as shown before, efficient IR absorbers (heaters), for example, graphite (Asbury Chemicals, Acid Washed Graphite Flakes), can be added to the gel, which increase the speed of heliotropic motion to reach a $70^{\circ}$ angle from $250 \mathrm{~s}$ in undoped gels to $125 \mathrm{~s}$ in doped gels without loss of reversibility, as shown in Figure 2d.

\section{Artificial nyctinasty through swelling/deswelling and transpiration}

In the design for nyctinasty, we have added two gel actuators at the leaf-stem junctions on the crease line marked
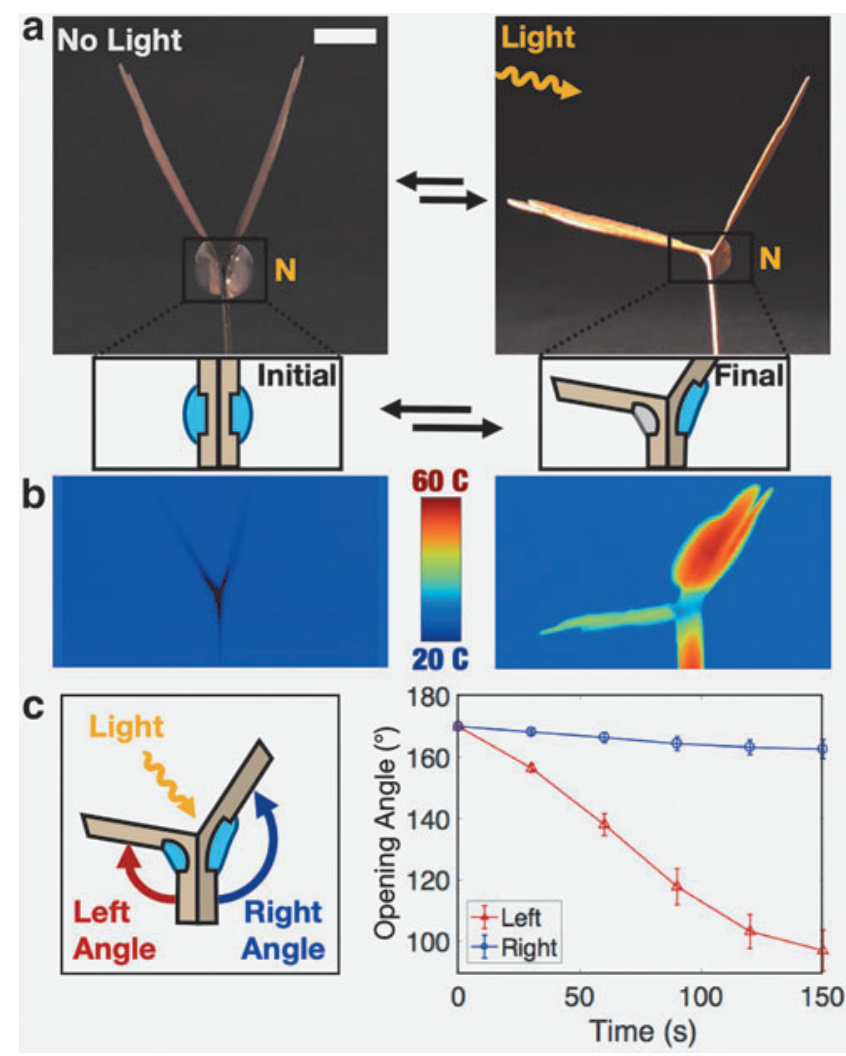

FIG. 3. The plant robot operated by transpiration from the hydrogel muscles showing nyctinasty. (a) Visible and (b) thermal images of the robot operation; when the plant in the dark steady state (left) is exposed to light, water evaporates faster from the hydrogel/paper muscle on the illuminated and warmer side, closer to the light source, the connected leaf opens toward the light source, until the leaf casts a shadow on the muscle. Scale bar $=1 \mathrm{~cm}$. (c) Opening angle of the left leaf compared with that of the right leaf upon illumination (direction of the light $=$ left , elevation angle $=45^{\circ}$ ). Color images are available online.

with $\mathrm{N}$ in Figure 2a. As shown in Figure 3 and Supplementary Movie S4, upon illumination, nyctinastic actuators are heated and contracted in a few minutes (similar to the heliotropic system in Fig. 2), the choice of which actuator is contracted more in comparison with the other one and the position at which the steady state forms depend on the intensity of the light source, its direction, and elevation. This can also be visualized in the plant garden (Fig. 1b; Supplementary Movie S2); for plants that are closer to the light source, the extent of leaf opening is much more pronounced than the plants farther away from the source. Finally, when the light is turned off, evaporation stops and the leaves close upon hydration and swelling of the actuators if the plant is connected to a water reservoir.

\section{Transpiration feedback}

Reversibility by rehydration can only be achieved in the plant robot through a connection of the plant roots to a water reservoir that serves as a continuous water source. The transpiration feedback through dehydration/rehydration cycles provides stabilization at a given metastable position in 
a
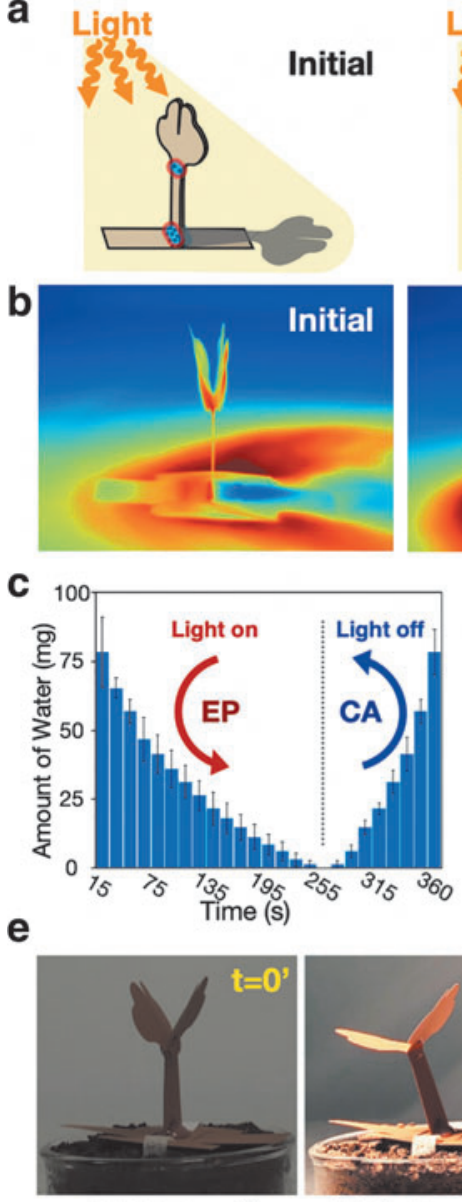

Light is off

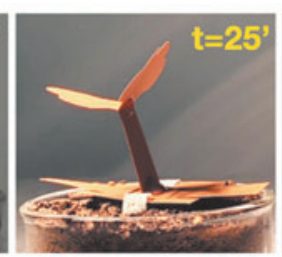

Heliotropism
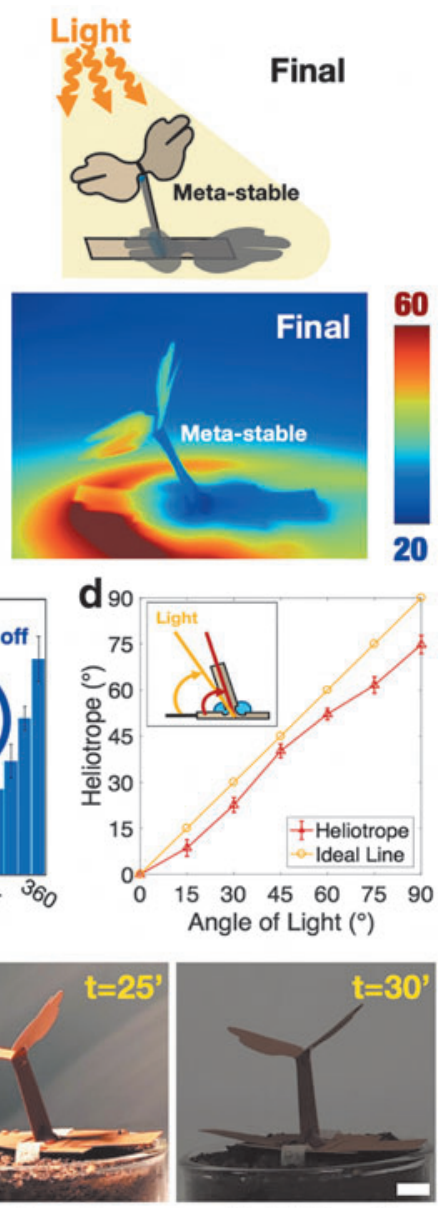

Light is off

FIG. 4. Self-regulated elevation tracking through material feedback in a plant displaying both heliotropism and nyctinasty. (a) Schematic representation and (b) thermal images of the initial and final (steady-state) positions, the latter is attained when the hydrogel muscle loses water and contracts upon light exposure. Any overturning is prevented since the light is blocked by the shadows cast by leaves of the plant on the muscle. (c) Changes in the amount of water in light (EP) and dark (CA) cycles. (d) The self-regulated heliotropic action of the plant [reflected in the average bending angle recorded at different elevation angles of light (red)] in comparison with an ideal heliotropic behavior (yellow). (e) The feedback is reversible up to 50 cycles, as shown in the photo and in Supplementary Movie S1. CA, capillary action; $\mathrm{EP}$, evaporation. Color images are available online.

heliotropic motion and therefore the continuous autonomous operation of the plant robot. As we described above, illuminating one of the gel actuators leads to its contraction and the bending of the stem toward the light source, which continues until the leaves (which also bend as a result of the stem motion) cast shadows on the hydrogel, as shown in Figure 4a and $b$. During illumination of the gel, the rate of evaporation is higher than the rate of water transport from the roots (connected to the reservoir) to the gel, as is evident from the decrease in weight of water during illumination (Fig. 4c).

However, as the shadow moves over to the gel and covers it, the rate of evaporation is lower than the rate of water transport from the roots, therefore the gel rehydrates and

swells as displayed in the weight increase of the plant (Fig. 4c). This action of swelling bends the stem away from the light source. However, then, the shadow also moves and the gel is illuminated again. This bending/straightening cycle provides the transpiration feedback, which keeps the plant in a steady state controlled solely by the elevation of the light source (Fig. 4d; Supplementary Movie S1). Shadow-cast stabilization and the feedback enable the plant to track a continuum of elevation angles with good precision (a comparison with the ideal heliotrope is provided in Fig. 4d), rather than an on-off behavior, which would have been observed if the shadow was absent.

In the design shown in Supplementary Movie S1, nyctinasty is not self-regulated on purpose (no connection between actuator $\mathbf{N}$ and the roots) to show the effect of shadow-casting feedback on the self-regulating heliotropism. A lower heliotropic bending angle at consecutive cycles is observed due

a
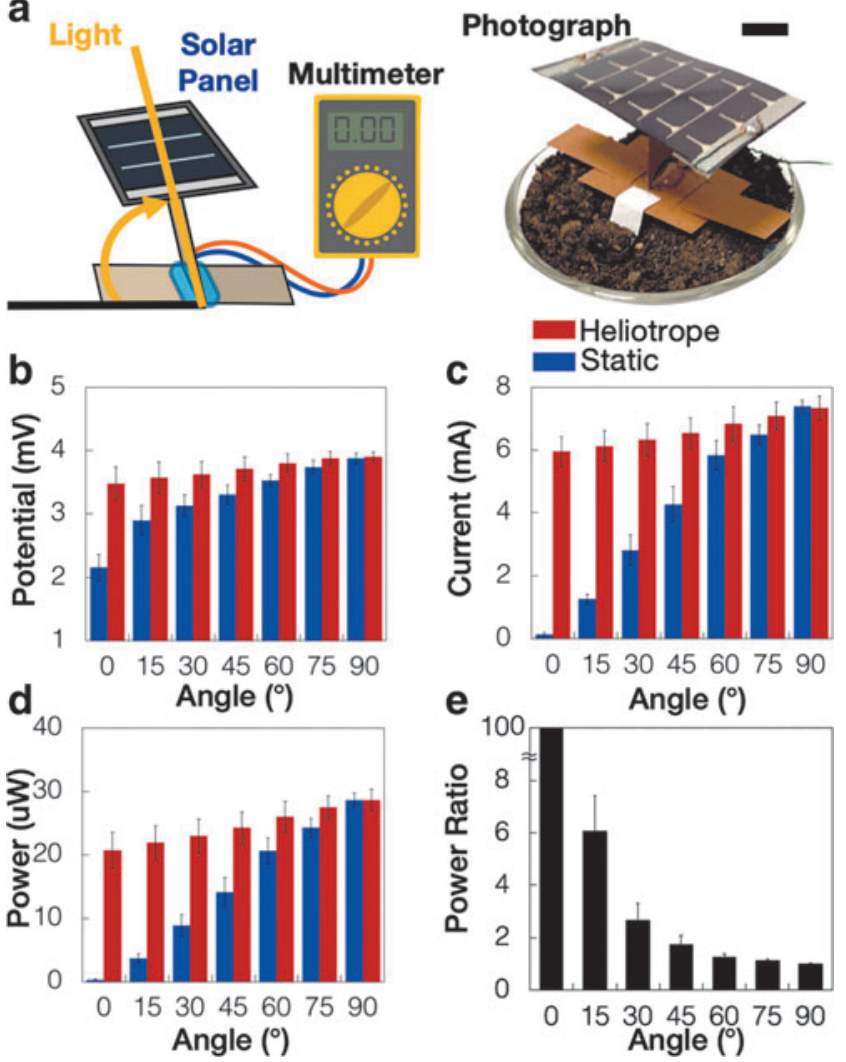

FIG. 5. Paper plant as a solar tracker. (a) The photo and schematic illustration of the self-regulated paper robot system, in which a thin solar panel (Flexible Solar Panel SP3-37; PowerFilm) is integrated into the paper plant robot. (b-d) Potential and current power (respectively) generated by solar panels upon illumination with the light source positioned at different elevation angles when the system is stationary (blue bars) versus when the system is heliotropic (red bars). (e) The ratio of the power generated by the heliotropic plant versus the power generated by a nonheliotropic plant (with no muscles and no feedback) at different elevation angles. The autonomous heliotrope harvests more solar energy than the nonheliotrope at lower elevation angles of the light source; for example, six times more power is obtained for the system operating at a light source elevation of $15^{\circ}$. Color images are available online. 
to the increase in the nyctinastic opening angle of the leaves. These feedback cycles were found to be fully reversible up to 30 cycles, the maximum number of cycles we have tried so far in our experiments.

\section{Discussion}

\section{Performance characteristics}

The paper plant robots can be made to perform as solar trackers by addition of a thin solar panel (Flexible Solar Panel SP3-37 from PowerFilm). The performance of the robot can be measured through the energy harvested by the solar panel leaves. Both the current and potential power levels generated by the solar panel increase with heliotropism (Fig. 5). The energy harvesting efficiency of the heliotrope (power generated by solar panels) therefore increases at lower elevation angles, for example, it can reach up to $\sim 600 \%$ (for $15^{\circ}$ elevation of the light source) of that of the nonheliotropic (static) plant, which has no actuators and lacks the mechanism for feedback.

Elevation tracking through self-regulation achieved by material feedback significantly increases the precision of solar tracking (Fig. 4d) in comparison with the existing examples of artificial heliotropes ${ }^{21}$ that operate only between not bent and fully bent states. It is not straightforward to compare the other performance characteristics of our system with others since these systems were not examined under identical conditions (e.g., with the same solar panels) and because the other systems lack elevation tracking. ${ }^{21}$

\section{Other designs that can be attained by artificial transpiration and material feedback}

Self-regulation through artificial transpiration in plant robotics can be extended to bioinspired plant parts. As a display of some of these possible designs, we show here stomatal opening/ closing behavior (Supplementary Fig. S11). In plants, stomatal opening is controlled by guard cells. In our artificial stomata, the control is through transpiration feedback attained by artificial actuators obtained by depositing hydrogels on the crease patterns of our origami designs. For selection and production of the origami design, please see Supplementary Data. The origami/kirigami structures can be extended to have complex architectures and functions, all of which can be operated upon (self-regulated) transpiration, as can be seen in the second part of the Supplementary Movie S5-a complex paper plant from a gift card was operated as an example.

The simple designs on cellulose/hydrogel systems we have shown successfully display the artificial feedback through transpiration by achieving stabilization at a metastable state that is regulated by the light input. In plants and other living organisms, self-regulation through biochemical feedback is vital for life. In artificial systems though, the applications of self-regulation were so far limited to a few, but important, examples, ${ }^{42-45}$ and in soft robotics, there was no such example so far. Achieving self-regulation in soft robots opens new possibilities for lightweight robots and miniaturization. It can also help to expand the material selection and designs that allow for incorporation of (bio)chemical reactions in soft permeable bodies that can power the robots. We hope to have shown that such feedback attained through careful choice of materials and geometrical design can help to achieve the embodied intelligence ${ }^{46}$ and much more in soft robotics.

\section{Acknowledgments}

The authors thank Asbury chemicals for the donation of graphite flakes.

\section{Authors' Contributions}

S.D.C. conducted the experiments. S.D.C., H.T.B., and B.B. conceived the idea, designed the experiments, and wrote the article.

\section{Author Disclosure Statement}

No competing financial interests exist.

\section{Funding Information}

This work was supported by FLAG-ERA Robocom++ and TÜBITTAK under award number 117M004. B.B. gratefully acknowledges the support from BAGEP 2016 (Science Academy Young Scientists Program) award.

\section{Supplementary Material}

Supplementary Data

Supplementary Figure S1

Supplementary Figure S2

Supplementary Figure S3

Supplementary Figure S4

Supplementary Figure S5

Supplementary Figure S6

Supplementary Figure S7

Supplementary Figure S8

Supplementary Figure S9

Supplementary Figure S10

Supplementary Figure S11

Supplementary Movie S1

Supplementary Movie S2

Supplementary Movie S3

Supplementary Movie S4

Supplementary Movie S5

\section{References}

1. Tolley MT, Shepherd RF, Mosadegh B, et al. A resilient, untethered soft robot. Soft Robot 2014;1:213-223.

2. Wehner M, Truby RL, Fitzgerald DJ, et al. An integrated design and fabrication strategy for entirely soft, autonomous robots. Nature 2016;536:451-455.

3. Park SJ, Gazzola M, Park KS, et al. Phototactic guidance of a tissue-engineered soft-robotic ray. Science 2016;353: $158-162$.

4. Li T, Li G, Liang Y, et al. Fast-moving soft electronic fish. Sci Adv 2017;3:e1602045.

5. Baytekin B, Cezan S, Baytekin HT, et al. Artificial heliotropism and nyctinasty based on optomechanical feedback and no electronics. Soft Robot 2017;5:1-7.

6. Terryn S, Brancart J, Lefeber D, et al. Self-healing soft pneumatic robots. Sci Robot 2017;2:eaan4268.

7. Sidorenko A, Krupenkin T, Taylor A, et al. Reversible switching of hydrogel-actuated nanostructures into complex micropatterns. Science 2007;315:487-490.

8. Zeng, Wani OM, Wasylczyk P, et al. Self-regulating iris based on light-actuated liquid crystal elastomer. Adv Mater 2017;29:1701814. 
9. Darwin C, Darwin F. The Power of Movements in Plants. London, UK: John Murray, 1880.

10. Argiolas A, Puleo GL, Sinibaldi E, et al. Osmolyte cooperation affects turgor dynamics in plants. Sci Rep 2016;6: 30139.

11. Must I, Sinibaldi E, Mazzolai B. A variable-stiffness tendrillike soft robot based on reversible osmotic actuation. Nat Commun 2019;10:1-8.

12. Mazzolai B, Mondini A, Corradi P, et al. A miniaturized mechatronic system inspired by plant roots for soil exploration. IEEE ASME Trans Mechatronics 2011;16:201-212.

13. Poulek V, Libra M. New low-cost tracking ridge concentrator. Sol Energy Mater Sol Cells 2000;61:199-202.

14. Sadeghi A, Tonazzini A, Popova L, et al. A novel growing device inspired by plant root soil penetration behaviors. PLoS One 2014;9:e90139.

15. Dicker MPM, Rossiter JM, Bond IP, et al. Biomimetic photo-actuation: sensing, control and actuation in suntracking plants. Bioinspir Biomim 2014;9:036015.

16. Häder DP, Lebert M. Photomovement. Amsterdam, The Netherlands: Elsevier, 2001.

17. Breger JC, Yoon C, Xiao R, et al. Self-folding thermomagnetically responsive soft microgrippers. ACS Appl Mater Interfaces 2015;7:3398-3405.

18. Malachowski K, Breger J, Kwag HR, et al. Stimuliresponsive theragrippers for chemomechanical controlled release. Angew Chem, Int Ed 2014;53:8045-8049.

19. Na JH, Evans AA, Bae J, et al. Programming reversibly selffolding origami with micropatterned photo-crosslinkable polymer trilayers. Adv Mater 2015;27:79-85.

20. Gustavsson PE, Larsson PO. Superporous agarose, a new material for chromatography. J Chromatogr A 1996;734: 231-240.

21. Li C, Liu Y, Huang X, et al. Direct sun-driven artificial heliotropism for solar energy harvesting based on a photothermomechanical liquid-crystal elastomer nanocomposite. Adv Funct Mater 2012;22:5166-5174.

22. Jamal M, Kadam SS, Xiao R, et al. Bio-origami hydrogel scaffolds composed of photocrosslinked PEG bilayers. Adv Healthc Mater 2013;2:1142-1150.

23. Laflin KE, Morris CJ, Muqeem T, et al. Laser triggered sequential folding of microstructures. Appl Phys Lett 2012; 101:131901.

24. Li S, Vogt S, Rus D, et al. Fluid-driven origami-inspired artificial muscles. Proc Natl Acad Sci 2017;114:13132-13137.

25. Martone PT, Boller M, Burgert I, et al. Mechanics without muscle: biomechanical inspiration from the plant world. Integr Comp Biol 2010;50:888-907.

26. Vandenbrink JP, Brown EA, Harmer SL, et al. Turning heads: the biology of solar tracking in sunflower. Plant Sci 2014;224:20-26.

27. Koller D. Solar navigation in plants. In: Hader DP, Lebert M (Eds). Photomovement. Amsterdam, The Netherlands: Elsevier, 2001, pp. 833-896.

28. Maurel C, Verdoucq L, Rodrigues O. Aquaporins and plant transpiration. Plant Cell Environ 2016;39:2580-2587.

29. Nobel PS. Physicochemical and Environmental Plant Physiology. London, UK: Elsevier, 2009.

30. Bar-On YM, Phillips R, Milo R. The biomass distribution on Earth. Proc Natl Acad Sci U S A 2018;115:6506-6511.
31. Jasechko S, Sharp ZD, Gibson JJ, et al. Terrestrial water fluxes dominated by transpiration. Nature 2013;496:347350 .

32. Huang G, Zhu Y, Liao Z, et al. Experimental investigation of self-pumping internal transpiration cooling. Int $\mathrm{J}$ Heat Mass Transf 2018;123:514-522.

33. Huang G, Zhu Y, Liao Z, et al. Biomimetic self-pumping transpiration cooling for additive manufactured porous module with tree-like micro-channel. Int $\mathrm{J}$ Heat Mass Transf 2019;131:403-410.

34. Taiz L, Zeiger E. Plant Physiology. Sunderland, UK: Sinauer Associates, Inc., 2010.

35. Klemm D, Heublein B, Fink HP, et al. Cellulose: fascinating biopolymer and sustainable raw material. Angew Chem Int Ed 2005;44:3358-3393.

36. Cui H, Du X, Wang J, et al. Poly(N-isopropylacrylamide) hydrogel-based shape-adjustable polyimide films triggered by near-human-body temperature. In: Proceedings of the Annual International Conference of the IEEE Engineering in Medicine and Biology Society, EMBS, October 2016, pp. 4197-4200.

37. Rich SI, Wood RJ, Majidi C. Untethered soft robotics. Nat Electron 2018;1:102-112.

38. Han D, Farino C, Yang C, et al. Soft robotic manipulation and locomotion with a 3D printed electroactive hydrogel. ACS Appl Mater Interfaces 2018;10:17512-17518.

39. Kim YS, Liu M, Ishida Y, et al. Thermoresponsive actuation enabled by permittivity switching in an electrostatically anisotropic hydrogel. Nat Mater 2015;14:1002-1007.

40. Morales D, Palleau E, Dickey MD, et al. Electro-actuated hydrogel walkers with dual responsive legs. Soft Matter 2014;10:1337-1348.

41. Ghasemi H, Ni G, Marconnet AM, et al. Solar steam generation by heat localization. Nat Commun 2014;5:4449.

42. Grinthal A, Aizenberg J. Adaptive all the way down: building responsive materials from hierarchies of chemomechanical feedback. Chem Soc Rev 2013;42:7072-7085.

43. He X, Aizenberg M, Kuksenok O, et al. Synthetic homeostatic materials with chemo-mechano-chemical selfregulation. Nature 2012;487:214-221.

44. Lagzi I, Kowalczyk B, Wang D, et al. Nanoparticle oscillations and fronts. Angew Chem Int Ed 2010;49:86168619.

45. Warren SC, Guney-Altay O, Grzybowski BA. Responsive and nonequilibrium nanomaterials. J Phys Chem Lett 2012; 3:2103-2211.

46. Pfeifer R, Lungarella M, Iida F. Self-organization, embodiment, and biologically inspired robotics. Science 2007; 318:1088-1093.

Address correspondence to: Bilge Baytekin

Department of Chemistry Bilkent University Ankara 06800 Turkey

E-mail: b-baytekin@fen.bilkent.edu.tr 